\title{
A $0.5 \%$ chlorhexidine gluconate in $70 \%$ isopropyl alcohol swab was more effective than 2 other methods for intravenous skin antisepsis
}

\author{
LeBlanc A, Cobbett S. Traditional practice versus evidence-based practice for IV skin preparation. Can J Infect Con \\ 2000; Spring:9-14. \\ QUESTION: Which of 3 methods for skin antisepsis before peripheral intravenous (IV) \\ therapy is most effective for preventing catheter related infection?
}

\section{Design}

Randomised \{allocation concealed\}*, blinded \{investigators, patients, outcome assessors $\}^{*}$, controlled trial with follow up at 72 hours after removal of the IV catheter.

\section{Setting}

A 139 bed, acute care hospital in Yarmouth, Nova Scotia, Canada.

\section{Patients}

300 patients who required a peripheral IV catheter and were able to read and understand English. Exclusion criteria was an IV catheter that remained in situ for $<8$ hours. Patients were recruited from the hospital's medical, surgical, intensive care, obstetrics and gynaecology, and outpatient and emergency services. Follow up was $81 \%$.

\section{Intervention}

Before peripheral IV insertion, patients were allocated to receive $1 \mathrm{swab}$ of $0.5 \%$ chlorhexidine gluconate (CHG) in 70\% isopropyl alcohol (group $1, \mathrm{n}=100$ ), a $70 \%$ isopropyl alcohol swab followed by a $10 \%$ povidone-iodine swab (group 2, $\mathrm{n}=100$ ), or a $10 \%$ povidone-iodine swab followed by a $70 \%$ isopropyl alcohol swab (group 3, $\mathrm{n}=100$ ).

\section{Main outcome measures}

Local catheter related infection (defined as $\geqslant 15$ colony forming units in the absence of accompanying clinical symptoms), probable catheter related infection (defined as having fever $\geqslant 38{ }^{\circ} \mathrm{C}$, pain, erythema, or heat at the involved vascular site and $>15$ colonies cultured from the intravascular cannula tip), and signs and symptoms at 72 hours after removal of the IV catheter.

\section{Main results}

Group 1 had a lower rate of probable catheter related infection than group $2\{\mathrm{p}=0.002\}^{\dagger}$ or group 3 $\{\mathrm{p}=0.01\}^{\dagger}$ (table). No differences existed among the 3 groups for rates of local catheter related infection. Fewer patients in group 1 had redness or pain at the IV site than those in groups 2 or $3\{p \leqslant 0.001\}^{\dagger}$. Fewer patients in group 1 had fever than those in group 3 $\{\mathrm{p}=0.01\}^{\dagger}$.

\section{Conclusion}

Patients who had skin antisepsis before peripheral intravenous therapy with $0.5 \%$ chlorhexidine gluconate had a lower risk of probable catheter related infection than those who had skin antisepsis with an alcohol swab followed by a povidone-iodine swab or a povidine-iodine swab followed by an alcohol swab.

*Information provided by author.

tp Value calculated from data in article.
Sources of funding: SoluMed Inc and Johnson and Johnson.

For correspondence: Ms A LeBlanc, Yarmouth Regional Hospital Operated by the Western Regional Health Board, 60 Vancouver Street, Yarmouth, NS B5A 2P5, Canada.Fax +1902 7420369 .

Comparison of 3 intravenous skin preparation methods on risk of probable catheter related infection at 72 hours after IV catheter removal $\$$

\begin{tabular}{llll} 
Comparisons & Event rates & RRR (95\% Cl) & NNT (CI) \\
CHG $v$ alcohol followed by povidone-iodine & $1.2 \% v 12.5 \%$ & $90 \%(44$ to 98$)$ & 9 (5 to 23) \\
\hline CHG $v$ povidone-iodine followed by alcohol & $1.2 \% v 9.9 \%$ & $88 \%(27$ to 98$)$ & 12 (6 to 48) \\
\hline fCHG $=$ chlorhexidine gluconate. Other abbreviations defined in glossary; & RRR, NNT, and Cl calculated from data
\end{tabular}

in article.

\section{COMMENTARY}

It is perhaps surprising that there is only limited research available on skin preparation before IV cannulation. Hospital acquired infection has important detrimental effects on patient comfort, morbidity, and mortality and increases length of hospital stay and healthcare costs. ${ }^{1}$ Thus, evidence that can help to reduce the incidence of infection has important implications for nursing practice.

This randomised controlled trial by LeBlanc and Cobbett compared 3 different methods of skin antisepsis before IV catheter insertion. The results suggest a reduction in probable catheter related infection in those patients whose skin was prepared using $0.5 \%$ chlorhexidine gluconate in $70 \%$ alcohol compared with alcohol and povidone iodine combinations. No differences existed between the groups in colonisation of the catheter tips.

The randomised design of this trial should ensure an even distribution among the groups of those factors likely to influence the outcomes, although the authors did not provide important baseline data that would convince us of this. For example, pain, heat, and erythema (used in this study as markers of infection) are also signs of non-specific phlebitis. Furthermore, we are not told how the method of catheter fixation (which may influence the incidence of phlebitis) was distributed across groups. ${ }^{2}{ }^{3}$ Siting of IV may also be important and UK nurses are taught that cannulation of the median cubital vein is more likely to cause irritation than cannulation of the superficial palmar or radial veins.

Therefore, although this study on its own does not provide evidence to change practice, it highlights the need for further primary research and perhaps a systematic review that synthesises research on skin preparation and catheter fixation in IV treatment.

Alun Roebuck, RN, BA Research Fellow, Department of Health Studies University of York York, UK

1 Hospital Infection Working Group. Hospital infection control. Guidance on the control of infection in hospitals. BAPS Health Publication Unit, 1995.

2 Campbell H, Carrington M. Peripheral IV cannula dressings: advantages and disadvantages. Br J Nurs 1999;8:1420-7.

3 Fewer patients dislodge peripheral intravenous catheters with transparent dressing than with gauze dressings [abstract]. Evidence-Based Nursing 1998;1:81. Abstract of: TripepiBova KA, Woods KD, Loach MC. Am J Crit Care 1997;6:377-81. 